\title{
Efeito da bebida de café descascado sobre a atividade antioxidante, os parâmetros hematológicos e bioquímicos em ratos
}

\author{
Peeled coffee brew effect in the antioxidant activity hematological and biochemical parameters in rats
}

\author{
Stella Maris da Silveira DUARTE ${ }^{1 *}$, Celeste Maria Patto de ABREU², Hillary Castle de MENEZES 3 , \\ Fernanda Borges de Araujo PAULA ${ }^{1}$, Rosemary Gualberto Fonseca Alvarenga PEREIRA ${ }^{4}$, \\ Cibele Marli Cação Paiva GOUVÊA ${ }^{5}$
}

\section{Resumo}

O objetivo deste estudo foi verificar o efeito da bebida filtrada, preparada com café-arábica descascado, sobre a peroxidação de lipídios, os parâmetros hematológicos e bioquímicos in vivo. Para isso foram utilizados ratos que ingeriram $280 \mathrm{mg} / \mathrm{kg} / \mathrm{dia}$ da bebida de café por 7 dias (tratamento agudo) e 30 dias (tratamento crônico). A fim de determinar se a bebida de café é capaz de reduzir o estresse oxidativo, foi analisada a peroxidação de lipídios isolados de cérebro de rato analisando-se as substâncias reativas do ácido tiobarbitúrico. A ingestão da bebida por 7 e 30 dias inibiu significativamente a peroxidação lipídica ( $\mathrm{p}<0,05$ e p < 0,001), respectivamente, em comparação ao controle. No entanto, não houve diferença significativa entre a porcentagem de inibição da peroxidação quando foram comparados os tratamentos agudo $(48,6 \%)$ e crônico $(53,4 \%)$. A ingestão crônica de doses moderadas da bebida filtrada de café não produziu modificação significativa no nível plasmático dos parâmetros bioquímicos e hematológicos analisados. Os resultados indicam que a ingestão moderada de café filtrado pode ter efeito benéfico para a saúde, por inibir a peroxidação lipídica e não interferir nos níveis séricos de colesterol e triglicerídeos em ratos. Palavras-chave: café; lipoperoxidação; colesterol; ácido úrico; triglicerídeos; parâmetros hematológicos.

\begin{abstract}
The aim of this study was to verify the effect of filtered coffee brew prepared with peeled Coffea arabica on the lipid peroxidation and on hematological and biochemical parameters in vivo. Rats were ingested $280 \mathrm{mg} / \mathrm{kg} /$ day of coffee brew for 7 days (acute treatment) and 30 days (chronic treatment). In order to determine whether coffee can reduce the oxidative stress, the rats brain isolated lipid peroxidation was accessed evaluating the thiobarbituric acid-reactive substances (TBARs). The ingestion of coffee brew for 7 and 30 days inhibited, significantly, the malondialdehyde concentration $(\mathrm{p}<0.05$ and $\mathrm{p}<0.001)$, respectively when compared to the control. However, there was no significantly difference $(\mathrm{p}>0.05)$ between the percentage of peroxidation inhibition when the acute $(48.6 \%)$ and chronic $(53.4 \%)$ treatments were compared. The chronic ingestion of moderate doses of filtered coffee brew did not modify the plasma level of the hematological and biochemical parameters analyzed. The results indicate the beneficial health effect of moderated filtered coffee brew ingestion since it inhibited lipid peroxidation, but did not change the rats cholesterol and triacylglycerol plasma levels.

Keywords: coffee; lipid peroxidation; cholesterol; uric acid; triacylglycerol; hematological parameters.
\end{abstract}

\section{Introdução}

A doença arterial coronariana (DAC) secundária à aterosclerose destaca-se nos dias atuais como a principal causa de morbidade e mortalidade nas sociedades industrializadas. Nos Estados Unidos foi responsável por um terço dos óbitos ocorridos nas últimas décadas. Dados brasileiros mostram que a situação no nosso meio é semelhante. Cerca de 31,0\% dos óbitos por causas determinadas ocorrem por doenças do aparelho circulatório (FUNDAÇÃO, 2004; SANTOS-FILHO; MARTINEZ, 2002; OLIVEIRA et al., 2006). A dislipidemia, condição na qual há concentrações anormais de lipídios ou lipoproteínas no sangue é um fator de risco importante para o desenvolvimento de complicações da aterosclerose. Em diferentes populações, estão bem estabelecidas as correlações entre o risco para DAC e concentrações séricas elevadas de colesterol total (CT), particularmente de lipoproteínas de baixa densidade (LDL-C), assim como concentrações reduzidas de lipoproteínas de alta densidade (HDL-C) (CASTELLI et al., 1986; MENOTTI et al., 2000; MARTINEZ et al., 2003). Na aterosclerose ocorre diminuição marcante dos mecanismos de defesa antioxidante (TESFAMARIAM, 1994; YOKOYAMA, 2004). Trabalhos têm correlacionado também o desenvolvimento de aterosclerose com o aumento da peroxidação lipídica

Recebido para publicação em 15/7/2008

Aceito para publicação em 8/7/2009 (002274)

${ }^{1}$ Departamento de Análises Clínicas, Universidade Federal de Alfenas - UNIFAL, Rua Gabriel Monteiro da Silva, 714, CEP 37130-000, Alfenas - MG,

E-mail: stellaunifal@yahoo.com.br

2 Departamento de Química, Universidade Federal de Lavras - UFLA, CEP 37200-000, Lavras - MG

${ }^{3}$ Universidade Estadual de Campinas - UNICAMP, Campinas-SP, CEP 13083-970, Brasil

${ }^{4}$ Laboratório de Grãos e Cereais, Departamento de Ciência dos Alimentos, Universidade Federal de Lavras - UFLA, CEP 37200-000, Lavras - MG, Brasil

${ }_{5}$ Departamento de Ciências Biológicas, Universidade Federal de Alfenas -UNIFAL, Rua Gabriel Monteiro da Silva, 714, CEP 37130000, Alfenas - MG

${ }^{*}$ A quem a correspondência deve ser enviada 
(NIGRIS et al., 2003; YOKOYAMA, 2004). As espécies reativas de oxigênio formam radicais peroxila que propagam a cadeia de peroxidação lipídica abstraindo átomos de hidrogênio para formar hidroperóxidos e novos radicais de carbono, levando à oxidação de muitas moléculas de ácidos graxos (JIALAL; GRUNDY, 1992; BARREIROS, DAVID, 2006).

O malonaldeído (MDA) é um aldeído de cadeia curta, sendo um dos compostos medidos pela reação com o ácido tiobarbitúrico (TBARS). A formação de malonaldeído ocorre pela decomposição dos hidroperóxidos lipídicos e sua concentração tem sido utilizada para estimar a intensidade da peroxidação lipídica em sistemas biológicos em células e tecidos (BONNES; GUÉRIN, 1992; BARREIROS; DAVID, 2006).

Os compostos antioxidantes protegem os sistemas biológicos contra os efeitos potencialmente danosos de reações destas espécies reativas de oxigênio com diversos alvos celulares. A peroxidação lipídica pode ser inibida por antioxidantes que interrompem a cadeia de peroxidação reagindo com os radicais peroxila ou alcoxila e, desta forma, gerando um hidroperóxido e um radical livre formado a partir do antioxidante. Uma alternativa para prevenir a lesão celular causada pela peroxidação lipídica é o aumento de antioxidantes endógenos através da ingestão de antioxidantes (FANG et al., 2002; FREDSTROM, 2002; VALKO et al., 2007).

Entre as substâncias presentes nos vegetais com atividade antioxidante, destacam-se os fenóis (MANCINI-FILHO, 2001) e podem oferecer proteção contra DAC. A principal classe de compostos fenólicos é composta por ácidos hidroxicinâmicos, encontrados em quase todas as plantas (HERRMANN, 1976; KUHNAU, 1976; OLTHOF et al., 2001), sendo o principal representante o ácido cafeico, que ocorre, em muitos alimentos, esterificado ao ácido quínico, sendo chamado de ácido clorogênico. O café é a principal fonte de ácido clorogênico da dieta humana (GARAMBONE; ROSA, 2007).

Apesar dos inúmeros trabalhos de pesquisa, o efeito cardiovascular do consumo de café para humanos é ainda controverso (RIKSEN et al., 2009). Urgert e Katan (1997) observaram aumento do colesterol plasmático com a ingestão da bebida de café e atribuíram este efeito ao conteúdo de diterpenos da amostra, caveol e cafestol. Christensen et al. (2001) demonstraram que a abstenção da bebida filtrada de café diminui o colesterol plasmático. Entretanto, Mursu et al. (2005) não detectaram aumento da peroxidação lipídica e concentração plasmática total de homocisteína em homens saudáveis não fumantes, após a ingestão por longo período de múltiplas doses ou de uma dose de café. A revisão de Ranheim e Halvorsen (2005), aparentemente, demonstra que a ingestão de quantidades moderadas de bebida filtrada não está associada a efeitos cardiovasculares deletérios.

O objetivo deste estudo foi verificar o efeito da bebida filtrada preparada com café-arábica descascado sobre a peroxidação de lipídio, os níveis plasmáticos de colesterol total e frações e sobre os parâmetros hematológicos em ratos.

\section{Material e métodos}

\subsection{Obtenção do café}

Amostras de café in natura (Coffea arabica), cv. Mundo Novo descascado (100 L), safra 2001/2002, peneira $17 / 18$, sem defeitos, foram cedidas por Ipanema Agro Indústria Ltda. (Alfenas, MG) e preparadas especialmente para este estudo. As amostras foram torradas em torrador de laboratório com capacidade de $1 \mathrm{~kg}$, em grau de torra escuro. O ponto de torração foi determinado visualmente. Em seguida, os grãos torrados foram moídos (moedor elétrico Raiar, modelo RA21) em granulometria fina ( $70 \%$ de retenção em peneira 20 ), empacotados em embalagens de polietileno/alumínio/ polipropileno seladas a vácuo e estocados a $-20^{\circ} \mathrm{C}$ até o preparo da bebida.

\subsection{Caracterização do café}

A cor do café torrado e moído foi determinada usando-se um colorímetro (Chromameter-2 Reflectance, Minolta, Osaka, Japan) acoplado a um processador de dados (OP-300). O instrumento foi padronizado contra um branco antes de cada leitura. A cor foi expressa em parâmetros da escala CIE L ¿ $^{\star} a^{*}$, $\mathrm{b}^{*}$. Foram determinados: o teor de sólidos solúveis segundo Maeztu et al. (2001) e polifenóis (GEORGE et al., 2005); o pH da bebida ( $\mathrm{n}=5)$; e, o teor de ácidos cafeoilquínicos (CQA) por cromatografia líquida de alta eficiência. Após a extração dos CQA (TRUGO; MACRAE, 1984), foi realizada a cromatografia utilizando-se um sistema Knauer Chance equipado com coluna de fase reversa RP-18, injetor com 100 uL de alça, detector de UV a $315 \mathrm{~nm}$ e integrador HP. O sistema utilizado foi isocrático com vazão constante de $1,0 \mathrm{ml} /$ minuto. A fase móvel consistiu de tampão citrato tripotássico $0,01 \mathrm{M}, \mathrm{pH} 2,5$ (DUARTE et al., 2005).

\subsection{Preparo da bebida de café}

Para o preparo da bebida, $10 \mathrm{~g}$ de café em pó foram colocados sobre um filtro de papel Whatman N. 3. Em seguida, $100 \mathrm{~mL}$ de água deionizada a $90^{\circ} \mathrm{C}$ foram vertidos sobre o pó (NICOLI et al., 1997).

\subsection{Delineamento e grupos experimentais}

O estudo in vivo foi realizado de acordo com os princípios éticos na experimentação animal adotados pelo Colégio Brasileiro de Experimentação Animal (COBEA), aprovado pelo comitê de ética em pesquisas animais da Universidade Federal de Alfenas, em reunião de 04/03/2005. Foram utilizados ratos machos Wistar (Rattus norvegicus) com $270 \pm 20$ g, obtidos no Biotério da UNIFAL-MG. Os animais foram mantidos em caixas de polietileno, recebendo água e ração comercial ad libitum e foram divididos em 4 grupos de 12 animais:

- C7: controle; os animais receberam veículo (água) por 7 dias consecutivos;

- T7: tratado; os animais receberam a bebida filtrada de café por 7 dias consecutivos (tratamento agudo); 
- C30: controle; os animais receberam veículo (água) por 30 dias consecutivos;

- T30: tratado; os animais receberam a bebida filtrada de café por 30 dias consecutivos (tratamento crônico).

O veículo e a bebida de café recém-preparada foram administrados aos animais por gavagem, uma vez ao dia, por 7 dias (tratamento agudo) e 30 dias (tratamento crônico). A concentração da bebida de café foi de $280 \mathrm{mg} / \mathrm{kg} /$ dia, equivalente ao consumo humano diário de 4 xícaras de $50 \mathrm{~mL}$ da bebida de café $(10 \%)$.

\subsection{Determinação da peroxidação lipídica}

O cérebro dos animais de todos os grupos experimentais foi removido, pesado e homogeneizado, em banho de gelo, com auxílio de homogeneizador de tecidos (Tecnal - modelo TE-100), após adição de um volume de tampão fosfato, $\mathrm{pH} 7,2$ equivalente a 4 vezes o peso fresco do tecido. O homogeneizado obtido foi centrifugado a $1000 \times \mathrm{g}$, por 10 minutos, a $4{ }^{\circ} \mathrm{C}$ e a concentração proteica foi determinada (BRADFORD, 1976).

A peroxidação lipídica foi determinada pela formação de substâncias reativas com o ácido tiobarbitúrico (TBARS) (BUEGE; AUST, 1978). Amostras de $500 \mu \mathrm{L}$, de homogeneizado de cérebro de ratos dos grupos controle e tratado, foram adicionadas a $500 \mu \mathrm{L}$ de ácido clorídrico a $25 \%$ (v/v) e $500 \mu \mathrm{L}$ de ácido tiobarbitúrico a $1 \%(\mathrm{p} / \mathrm{v})$ em hidróxido de sódio a $0,1 \mathrm{M} \mathrm{e} 45 \mu \mathrm{L}$ de BHT a $2 \%(\mathrm{p} / \mathrm{v})$. A mistura foi aquecida em água fervente por 10 minutos, resfriada em banho de gelo por 10 minutos e centrifugada a $900 \times$ g, por 15 minutos. As TBARS foram determinadas por $\mathrm{Abs}_{535}$ e a concentração foi obtida utilizando-se tetraetoxipropano como padrão (BROWN; KELLY, 1996). A atividade antioxidante foi calculada como porcentagem de inibição da peroxidação de lipídios (I\%), por comparação ao controle (Equação 1):

$$
I \%=\frac{A c-A t}{A c} \times 100
$$

em que: $\mathrm{A}_{\mathrm{C}}$ : a absorbância das amostras do grupo controle; $\mathrm{e}$ At: absorbância das amostras do grupo tratado.

\subsection{Determinação dos parâmetros bioquímicos e hematológicos}

Amostras de sangue venoso periférico foram coletadas dos animais dos grupos controle e tratado 30 dias, para determinação dos níveis plasmáticos de colesterol total e frações, triglicerídeos, ácido úrico e dos parâmetros hematológicos. Os parâmetros bioquímicos foram determinados por método enzimáticocolorimétrico utilizando-se kits comerciais (Bioclin).

Os parâmetros hematológicos foram obtidos automaticamente em contador eletrônico de células (Coulter T-890).

\subsection{Análise estatística}

Os dados obtidos foram submetidos à análise de variância e comparados pelo teste de Tukey ou teste $t$-Student, quando $\mathrm{p}<0,05$.

\section{Resultados e discussão}

Apesar dos inúmeros estudos, o efeito cardiovascular do consumo de café em humanos é ainda controverso. Isto pode ocorrer porque os trabalhos utilizam diferentes técnicas experimentais, incluindo diferentes tipos de processamento, beneficiamento, torração, moagem e preparo da bebida, o que determina uma ampla variação da composição química do café (RICHELLE et al., 2001; DEL CASTILHO et al., 2002; NIGRIS et al., 2003; VAN DAM, 2008). Assim, nesse estudo o grau de torra foi caracterizado e foram determinados o $\mathrm{pH}$ e a concentração de sólidos solúveis para a padronização das amostras utilizadas, bem como para possibilitar a utilização de amostras semelhantes em outros trabalhos.

A análise de cor revelou os seguintes valores, seguidos do desvio padrão, para a luminosidade $\left(L^{*}\right): 29,07 \pm 0,81$ e coordenadas de cromaticidade $\left(\mathrm{a}^{\star}\right):+10,28 \pm 0,19$ e $\left(\mathrm{b}^{\star}\right)$ : $1,42 \pm 0,32$. O pH da bebida foi de $5,14 \pm 0,04$. O conteúdo de polifenóis foi de 6,26\% (equivalentes de ácido tânico) e o teor de sólidos solúveis de 2,25 $\pm 0,05 \%$. O conteúdo de CQA na bebida foi de $0,392 \%$ para 3 -CQA; $0,388 \%$ para 4 -CQA; e $0,545 \%$ para 5 -CQA.

A ingestão de vegetais, sucos e chás, sabidamente, exerce uma série de efeitos benéficos à saúde. Parte desses efeitos pode ser atribuída à presença de substâncias antioxidantes, que auxiliam a equilibrar o estresse oxidativo, prevenindo a instalação e a progressão da aterosclerose, uma das principais causas da doença arterial coronariana (YOKOYAMA, 2004). Dentre as substâncias presentes nos vegetais com atividade antioxidante, destacam-se os fenólicos, importantes componentes do café. O café é uma bebida muito popular no mundo e uma das principais fontes de substâncias fenólicas da dieta. Tais substâncias apresentam atividade protetora das membranas contra o dano oxidativo (SILVA et al., 2005).

Observou-se que a ingestão de café induziu inibição significativa da peroxidação lipídica, tendo sido de 48,60\% no grupo com T7 (tratamento agudo) e de 53,40\% no grupo T30 (tratamento crônico), o que foi evidenciado pela diminuição da concentração de MDA, quando comparada à dos controles C7 e C30, respectivamente. Entretanto, não houve diferença significativa entre os valores obtidos nos grupos T7 e T30.

No presente trabalho verificou-se que tanto a ingestão aguda como a crônica da bebida filtrada de café diminuiu a peroxidação lipídica. Não houve diferença significativa entre os dois protocolos de tratamento, indicando que a atividade antioxidante máxima da bebida pode ser obtida com a ingestão por 7 dias. A diminuição da peroxidação lipídica pode ser atribuída, pelo menos em parte, à presença de substâncias com atividade antioxidante, presentes no café mesmo após a torração, como os ácidos cafeoilquínicos e polifenóis (DUARTE et al., 2005). Daglia et al. (2004) demonstraram também que o café torrado em grau escuro apresentou atividade antioxidante in vitro e ex vivo e esses autores atribuíram essa atividade, principalmente, ao ácido 5-O-cafeoilquínico, componente presente na bebida de café utilizada neste trabalho. Espécies tóxicas do oxigênio causam danos oxidativos aos componentes celulares, podendo facilmente iniciar a peroxidação dos lipídios 
da membrana levando ao acúmulo de peróxidos lipídicos. Os produtos de peroxidação e seus produtos secundários como malonaldeído (MDA) são altamente reativos, podendo reagir com substratos biológicos como proteínas, lipídios e DNA (BUEGE; AUST, 1978; VASCONCELOS et al., 2007). Assim, o consumo de café pode contribuir para a diminuição da peroxidação lipídica, diminuindo a instalação e progressão de lesões celulares e teciduais, como a desestabilização do ateroma (THELLE et al., 1987; PANAGIOTAKOS et al., 2003).

A ingestão crônica da bebida filtrada de café não produziu modificação no nível plasmático dos parâmetros bioquímicos analisados (Tabela 1).

Estudos realizados, tanto epidemiologicamente como experimentalmente, indicam que a ingestão frequente de café aumenta os níveis de colesterol no plasma (THELLE et al., 1983; WEUSTEN-VAN DER WOUW et al., 1994; JEE et al., 2001). Este fenômeno foi primeiramente observado na Suécia, onde se encontrou forte correlação entre o consumo de café e o aumento de colesterol (THELLE et al., 1983). Deve-se ressaltar que os escandinavos são os maiores consumidores de café do mundo (TRUGO, 2001). Estudos comprovaram que o aumento de colesterol se deve a ingestão de cafestol e caveol, ambos presentes na fração lipídica do café (TERPSTRA et al., 2000; BOEKSCHOTEN et al., 2005). Quando o café é filtrado, a pequena fração lipídica que permanece na superfície da bebida aquosa é totalmente retida no filtro. Os povos escandinavos preferem o café turco, no qual o café moído é fervido em água e simplesmente decantado, antes do consumo. Na maioria dos países, o hábito predominante é o consumo de café filtrado o que explica a ocorrência desse fenômeno, principalmente, naquela região (TRUGO, 2001). Neste trabalho não houve aumento do nível de triglicerídeos, colesterol sérico e frações em ratos tratados com a bebida filtrada de café. Na patogênese da aterosclerose, a lipoproteína de alta densidade (HDL) apresenta um importante papel protetor, enquanto o aumento do colesterol, à custa de lipoproteína de baixa densidade (LDL), promove a aterogênese. O estudo realizado por Ito et al. (1998) também não encontrou alterações significativas nos níveis séricos de colesterol, triglicérides, HDL, em homens após a ingestão de 5 copos de café por dia, durante quatro semanas. Yukawa et al. (2004) demonstraram redução do colesterol total e MDA, enquanto os níveis séricos de triglicerídeos e HDL-colesterol não apresentaram alterações significativas após a ingestão por 4 semanas de $150 \mathrm{~mL}$ da bebida de café preparada com $8 \mathrm{~g}$ de café-arábica em $150 \mathrm{ml}$ de água fervendo. De acordo com McAnlis et al. (1998), o café não afeta o nível

Tabela 1. Parâmetros bioquímicos determinados no soro de rato controle (C30) e tratado por 30 dias com a bebida filtrada de café $(280 \mathrm{mg} / \mathrm{kg} / \mathrm{dia}$; T30) $(\mathrm{n}=12)$.

\begin{tabular}{lcc}
\hline \multicolumn{1}{c}{ Parâmetros } & C30 & T30 \\
\hline Colesterol total (mg/dL) & $84,6 \pm 18,7$ & $74,8 \pm 19,2$ \\
Colesterol-HDL (mg/dL) & $25,96 \pm 2,8$ & $24,48 \pm 2,1$ \\
Triglicerídeos (mg/dL) & $107,6 \pm 40,9$ & $92,4 \pm 51,4$ \\
Ácido úrico (mg/dL) & $1,48 \pm 0,76$ & $1,11 \pm 0,54$ \\
\hline
\end{tabular}

Todos os valores foram expressos como média \pm desvio padrão. Não houve diferença significativa entre os grupos, quando comparados pelo teste t-Student. de lipídios e susceptibilidade de oxidação de LDL, indicando que o consumo de café pode proteger contra a aterosclerose. Riksen et al. (2009) em seu trabalho de revisão também não encontraram associação entre o consumo de café e a incidência de aterosclerose. Assim, os resultados deste trabalho indicam que a ingestão de café pode contribuir para a prevenção da instalação e progressão da aterosclerose, pois aumenta a atividade antioxidante endógena.

Oácido úrico, um antioxidante endógeno (CHATTERJEE etal., 2005; VASCONCELOS et al., 2007), não apresentou variação da concentração plasmática, nos animais tratados com bebida de café. Tem sido descrito, no entanto, o aumento de ácido úrico no plasma, após a ingestão de bebidas ou alimentos ricos em fenóis (NATELLA et al., 2002). Contudo, Choi e Curhan (2007) observaram redução de ácido úrico, após a ingestão de café de maneira dose dependente.

Não foram observadas alterações significativas dos parâmetros hematológicos nos animais tratados com a bebida filtrada de café, quando comparados ao grupo controle e não há sinais de redução de hemoglobina, o que indicaria anemia (Tabela 2). Resultados semelhantes foram obtidos por Maeng et al. (2004).

Estudos envolvendo a progressão da aterosclerose têm se intensificado nos últimos anos, em função do aumento da incidência de doenças cardiovasculares, decorrentes da primeira. A progressão da aterosclerose aparece ligada ao estresse oxidativo e a patologias como infarto do miocárdio, acidentes vasculares, entre outros problemas (YOKOYAMA, 2004). Isto justifica a investigação de substâncias naturais com atividade antioxidante que podem contribuir para prevenção e progressão deste mal.

As pesquisas para seleção e triagem de substâncias presentes nos vegetais com atividade antioxidante têm sido conduzidas em animais de laboratório. Nesse contexto, os resultados do presente trabalho indicam que a ingestão diária moderada de café parece ser benéfica e pode contribuir para a diminuição da ocorrência e progressão da aterosclerose. Cabe ressaltar que, apesar de muito popular, o tipo de processamento, preparo da

Tabela 2. Parâmetros hematológicos de ratos controle (C30) e tratado por 30 dias com a bebida filtrada de café $(280 \mathrm{mg} / \mathrm{kg} / \mathrm{dia}$; T30) $(\mathrm{n}=12)$.

\begin{tabular}{lcr}
\hline \multicolumn{1}{c}{ Parâmetros } & C30 & \multicolumn{1}{c}{ T30 } \\
\hline Hemácias $\left(10^{6} / \mathrm{mm}^{3}\right)$ & $7,30 \pm 0,60$ & $7,30 \pm 0,50$ \\
Hemoglobina $(\mathrm{g} / \mathrm{dL})$ & $14,46 \pm 1,70$ & $14,42 \pm 1,20$ \\
Hematócrito $(\%)$ & $43,15 \pm 5,80$ & $42,57 \pm 3,20$ \\
VCM (fL) & $56,90 \pm 3,40$ & $56,80 \pm 2,40$ \\
HCM (pg) & $19,47 \pm 0,80$ & $19,76 \pm 0,70$ \\
CHCM (\%) & $34,15 \pm 1,50$ & $33,70 \pm 0,90$ \\
Granulócitos $\left(10^{3} / \mathrm{mm}^{3}\right)$ & $5,22 \pm 8,10$ & $5,30 \pm 0,10$ \\
Monócitos $(\%)$ & $13,04 \pm 5,00$ & $12,83 \pm 4,40$ \\
Linfócitos $(\%)$ & $71,60 \pm 11,00$ & $72,64 \pm 9,20$ \\
Plaquetas $\left(10^{3} / \mathrm{mm}^{3}\right)$ & $746,30 \pm 114,80$ & $720,54 \pm 121,86$ \\
\hline
\end{tabular}

Todos os valores foram expressos como média \pm desvio padrão. $\mathrm{n}=15$. Não houve diferença significativa entre os grupos, quando comparados pelo teste t-Student. 
bebida e o modo de consumo estão associados com a cultura e hábitos sociais próprios de cada país (NEHLIG; DEBRY, 1994) o que pode determinar a variação da composição e, portanto, das propriedades antioxidantes da bebida de café.

\section{Conclusão}

A partir dos resultados obtidos pode-se concluir que a ingestão de café filtrado diminui a peroxidação lipídica e não induz alteração de parâmetros hematológicos e bioquímicos em animais de laboratório. Assim, a ingestão moderada de café filtrado pode ter efeito benéfico para a saúde, contribuindo para diminuição da ocorrência e progressão da aterosclerose.

\section{Agradecimentos}

À Ipanema Agro-Indústria, pelo fornecimento do café.

À FAPEMIG, pela concessão de bolsa Jovem Doutor.

\section{Referências bibliográficas}

BARREIROS, L. B. S.; DAVID, J. M. Estresse oxidativo: relação entre geração de espécies reativas e defesa do organismo. Quimica Nova, v. 29, p. 113-123, 2006.

BOEKSCHOTEN, M. V. et al. Coffee oil consumption increases plasma levels of 7 alpha-hydroxy-4-cholesten-3-one in humans. Journal of Nutrition, v. 135, p. 785-799, 2005.

BONNES, T.; GUÉRIN, T. Is malonaldeyde a valuable of peroxidation? Biochemical Pharmacology, v. 44, n. 5, p. 985-988, 1992.

BRADFORD, M. M. A rapid and sensitive method for the quantification of microgram quantities of protein utilizing the principle of proteindye binding. Analytical Biochemistry, v. 72, p. 248-254, 1976.

BROWN, R. K.; KELLY, F. J. Peroxides and other products. In: PUNCHARD, N. A.; KELLY, F. J. (Ed.). Free radicals: a practical approach. New York: IRL Press, 1996. p. 119-131.

BUEGE, J. A.; AUST, S. D. Microsomal lipid peroxidation. Methods in Enzymology, v. 52, p. 302-310, 1978.

CASTELLI, W. P. et al. Incidence of coronary heart disease and lipoprotein cholesterol levels: the framingham study. Journal of the American Medical, v. 256, p. 2835-8, 1986.

CHATTERJEE, S. et al. A modified, economic, sensitive method for measuring total antioxidant capacities of human plasma and natural compounds using Indian saffron (Crocus sativus). Clinica Chimica Acta, v. 52, p. 155-163, 2005.

CHOI, H. K.; CURHAN, G. Coffee, tea, and caffeine consumption and serum uric acid level: the third national health and nutrition examination survey. Arthritis \& Rheumatism, v. 57, n. 5, p. 816-821, 2007.

CHRISTENSEN, B. et al. Abstention from filtered coffee reduces the levels of homocysteine and cholesterol - a randomized, controlled trial. American Journal of Clinical Nutrition, v. 74, p. 302-307, 2001.

DAGLIA, M. et al. In vitro e ex vivo antihydroxyl radical activity of green and roasted coffee. Journal of Agricultural and Food Chemistry, v. 5, p. 1700-1704, 2004.

DEL CASTILLO, M. D.; AMES, J. M.; GORDON, M. H. Effect of roasting on the antioxidant activity of coffee brews. Journal of Agricultural and Food Chemistry, v. 50, p. 3698-3703, 2002.
DUARTE, S. M. S. et al. Effect of processing and roasting of the antioxidant activity of coffee brews. Ciência e Tecnologia de Alimentos, v. 25, n. 2, p. 387-393, 2005.

FANG, Y. Z.; YANG, S.; WU, G. Free radicals, antioxidants, and nutrition. Nutrition, v. 18, p. 872-879, 2002.

FREDSTROM, S. Nitric oxide, oxidative stress, and dietary antioxidants. Nutrition, v. 18, p. 537-539, 2002.

BRASIL. Ministério da Saúde. Fundação Nacional da Saúde. Brasília, DF, c1999. Disponível em: <http://www.funasa.gov.br >. Acesso em: 16 mar. 2004.

GARAMBONE, E.; ROSA, G. Possíveis benefícios do ácido clorogênico à saúde. Alimentos e Nutrição, v. 18, p. 229-235, 2007.

GEORGE, S. et al. Rapid determination of polyphenols and vitamin $\mathrm{C}$ in plant-derived products. Journal of Agricultural and Food Chemistry, v. 53, n. 5, p. 1370-1373, 2005.

HERRMANN, K. Flavonols and flavones in food plants: a review. Food Technology, v. 11, p. 433-438, 1976.

ITO, T. et al. Effect of filtered coffee intake on lipid metabolism. European Journal of Clinical Nutrition, v. 52, p. 210-216, 1998.

JEE, S. H. et al. Coffee consumption and serum lipids: a meta-analysis of randomized controlled clinical trials. Am. J. Epidemiol., v. 153, p. 353-362, 2001.

JIALAL, I.; GRUNDY, S. D. Influence of antioxidant vitamins on LDL oxidation. Annals of the New York Academy of Sciences, v. 669, p. 239-248, 1992.

KUHNAU, J. The flavonoids. A class of semi-essential food components: their role in human nutrition. World Nutrition Reviews, v. 24, p. 117-191, 1976.

MAENG, S. H. et al. Micronuclei induction by 13 week-inhalation of 1,1-dichoro-1-fluorethane in Sprague-Dawley rats. Toxicology Letters, v. 146, p. 129-137, 2004.

MAEZTU, L. et al. Multivariate methods for characterization and classification of espresso coffees from different botanical varieties and types of roast by foam, taste, and mouth feel. Journal of Agricultural and Food Chemistry, v. 49, n. 10, p. 43-47, 2001.

MANCINI-FILHO, J. Importância química e nutricional dos antioxidantes naturalmente presentes nos alimentos. In: MERCADANTE, A. Z. et al. (Ed.). Ciência de alimentos: avanços e perspectivas. Campinas: FEA/UNICAMP, 2001. v. 2, p. 174-176.

MARTINEZ, T. L. R. et al. Campanha nacional de alerta sobre o colesterol elevado: determinação do nível de colesterol de 81.262 brasileiros. Arquivos Brasileiros de Cardiologia, v. 80, p. 631-634, 2003.

McANLIS, G. T. et al. Black tea consumption does not protect low density lipoprotein from oxidative modification. European Journal of Clinical Nutrition, v. 52, p. 202-206, 1998.

MENOTTI, A. et al. Coronary heart disease incidence in northern and southern European population: a reanalysis of seven countries study for a European coronary risk chart. Heart, v. 84, p. 238-44, 2000.

MURSU, J. et al. The effects of coffee consumption on lipid peroxidation and plasma total homocysteine concentrations: a clinical trial. Free Radical Biology \& Medicine, v. 38, p. 527-534, 2005.

NATELLA, F. et al. Coffee drinking influences plasma antioxidant capacity in humans. Journal and Agricultural and Food Chemistry, v. 50, p. 6211-6216, 2002. 
NEHLIG, A.; DEBRY, G. Potential genotoxic, mutagenic and antimutagenic effects of coffee: a review. Mutation Research, v. 317, p. 145-162, 1994.

NICOLI, M. C. et al. Antioxidant properties of coffee brews in relation to the roasting degree. Lebensmittel-Wissenschaft undTechnologie, v. 30, p. 292-297, 1997.

NIGRIS, F. et al. Oxidation-sensitive mechanisms, vascular apoptosis and atherosclerosis. Trends in Molecular Medicine, v. 9, p. 351-359, 2003.

OLIVEIRA, G. M. M. et al. Mortalidade por doenças cardiovasculares em três estados do Brasil de 1980 a 2002. Rev Panam Salud Publica, v. 19, p. 85-93, 2006.

OLTHOF, R. M.; HOLLMAN, C. H.; KATAN, B. M. Chlorogenic acid and caffeic acid are absorbed in humans. Journal of Nutrition, v. 131, p. 66-71, 2001.

PANAGIOTAKOS, D. B. et al. The J-Shaped effect of coffee consumption on the risk of developing acute coronary syndromes: the CARDIO2000 case-control study. Journal of Nutrition, v. 133, p. 28-32, 2003.

RANHEIM, T.; HALVORSEN, B. Coffee consumption and human health - beneficial or detrimental? - Mechanisms for effects of coffee consumption on different risk factors for cardiovascular disease and type 2 diabetes mellitus. Molecular Nutrition \& Food Research, v. 49, p. 274-284, 2005.

RICHELLE, M.; TAVAZZI, I.; OFFORD, E. Comparison of the Antioxidant activity of commonly consumed polyphenolic bverages (coffee, cocoa, and tea) prepared per cup. Journal and Agricultural and Food Chemistry, v. 49, p. 3438-3442, 2001.

RIKSEN, N. P.; RONGEN, G. A.; SMITS, P. Acute and long-term cardiovascular effects of coffee: implications for coronary heart disease. Pharmacology \& Therapeutics, v. 121, p. 185-191, 2009.

SANTOS-FILHO, R. D.; MARTINEZ, T. L. R. Fatores de risco para doença cardiovascular: velhos e novos fatores de risco, velhos problemas! Arquivos Brasileiros de Endocrinologia \& Metabologia, v. 46, p. 212-214, 2002.

SILVA, C. G. et al. Evaluation of antioxidant activity of Brazilian plants. Pharmacological Research., v. 52, p. 229-33, 2005.
TERPSTRA, A. H. M. et al. The hypercholesterolemic effect of cafestol in coffee oil in gerbils and rats. Journal of Nutritional Biochemistry, v. 11, p. 311-317, 2000.

TESFAMARIAM, B. Free radicals in diabetic endothelial cell dysfunction. Free Radical Biology \& Medicine, v. 16, p. 383-391, 1994.

THELLE, D. S.; HEYDEN, S.; FODOR, J. G. Coffee and cholesterol in epidemiological and experimental studies. Atherosclerosis, v. 67, p. 97-103, 1987.

TRUGO, L. C. Café: composição química e potencial nutracêutico. In: SIMPÓSIO LATINO AMERICANO DE CIENCIAS DE ALIMENTOS, 2001, Campinas. Anais... Campinas: Adriana Z. Mercadante, 2001. 272p.

TRUGO, L. C.; MACRAE, R. A study of the effect of roasting on the chlorogenic acid composition of coffee using HPLC. Food Chemical, v. 15, p. 219-227, 1984.

URGERT, R.; KATAN, B. The cholesterol-raising factor from coffee beans. Annual Review of Nutrition, v. 17, p. 305-324, 1997.

VALKO, M. et al. Free radicals and antioxidants in normal physiological functions and human disease. The International Journal of Biochemistry \& Cell Biology, v. 39, p. 44-84, 2007.

VAN DAM, R. M. Coffee consumption and risk of type 2 diabetes, cardiovascular diseases, and cancer. Appl. Physiol. Nutr. Metab., v. 33, p. 1269-1283, 2008.

VASCONCELOS, S. M. L. et al. Espécies reativas de oxigênio e de nitrogênio, antioxidantes e marcadores de dano oxidativo em sangue humano: principais métodos analíticos para sua determinação. Química Nova, v. 30, n. 5, p. 1323-1338, 2007.

WEUSTEN-VAN DER WOUW, M. P. et al. Identity of the cholesterolrising factor from boiled coffee and its effects on liver function enzymes. J. Lipid Res., v. 35, p. 721-733, 1994.

YOKOYAMA, M. Oxidant stress and atherosclerosis. Current Opinion in Pharmacology, v. 4, p. 110-115, 2004.

YUKAWA, G. S. et al. Effects of coffee consumption on oxidative susceptibility of low-density lipoproteins and serum lipid levels in humans. Biochemistry, v. 69, p. 70-74, 2004. 